\title{
Anti-biofouling $\mathrm{NH}_{3}$ gas sensor based on reentrant thorny $\mathrm{ZnO} /$ graphene hybrid nanowalls
}

\author{
Tian Hang ${ }^{1}$, Jiangming Wu', Shuai Xiao', Baohong $\mathrm{Li}^{1}$, Hongbo Li ${ }^{1}$, Chengduan Yang ${ }^{1}$, Cheng Yang ${ }^{1}$, Ning Hu (D)',
} Yonghang $\mathrm{Xu}^{2}$, Yu Zhang ${ }^{1}$ and $\mathrm{Xi}$ Xie $\mathbb{B}^{1,3}$

\begin{abstract}
Since toxic gas leakage may cause ecological environmental problems and even life-threatening damage, effective monitoring of toxic gas is of great importance and subject to increasing demand. However, complicated environmental factors, as well as various coexisting interferences can easily affect the sensitivity and selectivity of gas sensors, hindering their performance. Recent reports have successfully demonstrated the development of hierarchical nanostructures with desirable self-cleaning properties, yet gas sensors that can resist contamination have rarely been realized. Here, we developed a reentrant thorny $\mathrm{ZnO} /$ graphene hybrid nanowall structure that simultaneously repels liquid contamination and possesses $\mathrm{NH}_{3}$ gas sensing properties. The unique reentrant and hierarchical structure, featuring an interconnected vertical graphene nanowall framework with numerous $\mathrm{ZnO}$ nanospikes branched on the top nanowall, is highly repellent to liquids, even biofluids with low surface tension. The hierarchical structure consisting of gas sensing graphene and $\mathrm{ZnO}$ can be successfully applied as an $\mathrm{NH}_{3}$ gas sensor at room temperature, exhibiting not only excellent sensitivity, selectivity, and repeatability, but also outstanding stability even after bacterial contamination. This study provides a versatile method for fabricating reentrant and hierarchical structures with excellent liquid repellency, and offers a promising method for designing reliable gas sensors with anti-biofouling properties.
\end{abstract}

\section{Introduction}

Toxic gases, such as ammonia $\left(\mathrm{NH}_{3}\right)$, originating from exhaust gases in a variety of farming and industrial processes, including pharmaceutical and fertilizer manufacturing, can cause ecological environmental problems and life-threatening conditions ${ }^{1,2}$. For example, exposure to an $\mathrm{NH}_{3}$ concentration of $25 \mathrm{ppm}$ can lead to skin, eye, and lung irritation in the human body ${ }^{3}$. Prolonged exposure to high concentrations of toxic gases can even endanger human life, and thus, it is of great importance to

\footnotetext{
Correspondence: Yu Zhang (stszhyu@mail.sysu.edu.cn) or Xi Xie (xiexi27@mail. sysu.edu.cn)

${ }^{1}$ State Key Laboratory of Optoelectronic Materials and Technologies, School of Electronics and Information Technology, Guangdong Province Key Laboratory of Display Material and Technology, Sun Yat-Sen University, Guangzhou 510006, China

${ }^{2}$ School of Materials Science and Energy Engineering, Foshan University, Foshan 528000, China

Full list of author information is available at the end of the article

These authors contributed equally: Tian Hang, Jiangming Wu
}

monitor their concentrations. The emergence of gas sensors has enabled the conversion of chemical information about a specified gas into analytical signals so that the concentrations of toxic gases can be accurately detected and evaluated. If excessive amounts of toxic gases are present, an alarm associated with the gas sensor can be triggered. Recently, graphene has been demonstrated to have unique properties, including high charge-carrier mobility, a large surface area, and high thermal stability, making it a highly promising material for gas sensing applications, especially for detecting nitrogen-based gases $^{2,4-7}$. However, certain limitations, such as long recovery time and irreversibility, have hindered the further application of graphene in robust gas sensors ${ }^{8}$. Metal oxides (MOs), such as $\mathrm{ZnO}, \mathrm{SnO}$, and $\mathrm{WO}$, are also widely studied and utilized as gas sensing materials for toxic gas monitoring because of their advantages, including low cost and versatile design ${ }^{3,9-13}$. To date, many MO gas sensors have been introduced and have

\section{(c) The Author(s) 2020}

(c) (i) Open Access This article is licensed under a Creative Commons Attribution 4.0 International License, which permits use, sharing, adaptation, distribution and reproduction c. in any medium or format, as long as you give appropriate credit to the original author(s) and the source, provide a link to the Creative Commons license, and indicate if changes were made. The images or other third party material in this article are included in the article's Creative Commons license, unless indicated otherwise in a credit line to the material. If material is not included in the article's Creative Commons license and your intended use is not permitted by statutory regulation or exceeds the permitted use, you will need to obtain permission directly from the copyright holder. To view a copy of this license, visit http://creativecommons.org/licenses/by/4.0/. 
greatly promoted the progression of gas sensing technology, yet these sensors are still limited by their unsatisfactory sensitivity and selectivity, inherent high resistance, and relatively high working temperature ${ }^{14}$. To compensate for the deficiency of a single material alone, gas sensors based on hybrid materials developed by the integration of graphene and $\mathrm{MO}$ semiconductors have been proposed ${ }^{14-17}$. For example, enhanced gas sensing properties of $\mathrm{SnO}_{2}$ /graphene composites at room temperature (RT) have been reported ${ }^{18}$. High-performance $\mathrm{NH}_{3}$ and $\mathrm{NO}_{2}$ sensors based on $\mathrm{ZnO}$ nanostructures, and reduced graphene oxide (rGO) composites have also each been demonstrated ${ }^{15,16}$.

Despite this progress, accurate detection of toxic gases still presents challenges due to the complicated air composition in real environments, and potential fouling or damage to sensors by the environment. For example, in poultry farms, $\mathrm{NH}_{3}$ emissions can be highly concentrated due to the confined animal production in small and limited geographical areas ${ }^{19}$. Accurate estimates of $\mathrm{NH}_{3}$ emissions are essential but difficult to obtain under commercial poultry house conditions. Coexisting gases, as well as interferents, including water vapor, solid particles, dust, and microorganisms in the air, can significantly affect the detection accuracy and performance of gas sensors. Once these undesired interferents are adsorbed or attached on the active material of the sensing device, the underlying sensing functionality of the gas sensor can be inhibited ${ }^{20}$. Merely increasing the surface roughness or specific surface area is not sufficient for building a stable and persistent sensing interface, although such changes may improve the sensitivity ${ }^{21}$. To date, some antibiofouling biosensors with biocompatible or selfcleaning chemical coatings have been reported to provide improved sensing in liquid environments without being contaminated; however, the development of reliable gas sensors with resistance to contaminants in air or gas environments is still highly challenging, yet in great demand. In addition, superhydrophobic chemical coatings used for self-cleaning can generally passivate the sensing materials, presenting a barrier that may reduce the direct access of target molecules to the sensing materials, and thus potentially compromise the sensitivity or specificity of the sensor. The development of anti-biofouling sensors without additional chemical coatings has rarely been achieved.

Topographical features play an important role in the interaction between gases and surfaces ${ }^{15,22}$. Special geometries, such as hierarchical and reentrant structures, have shown favorable antifouling properties ${ }^{23-25}$. Such structures can form a composite solid-liquid-air interface that can effectively prevent liquids from penetrating and reduce the nonspecific adsorption of interfering substances ${ }^{23,26}$. Although superhydrophobic or superomniphobic chemical coatings can effectively prevent the adhesion of liquids or contaminants, passivation of the sensor surface by the chemical coating would block the sensor and interfere with sensing functionalities ${ }^{27}$. On the other hand, unlike traditional chemical modification approaches that require lowsurface-energy reagents (e.g., fluorine-containing coatings), reentrant structures have been reported to possess excellent antifouling properties even without low-surface-energy coatings $^{24,28}$. Antifouling reentrant structures are promising candidates for applications in designing functional sensors because the surface chemical properties will not undergo subsequent chemical modification, resulting in minimal changes in sensing functionalities.

In this article, we have developed a reentrant thorny $\mathrm{ZnO} /$ graphene hybrid nanowall structure (denoted as GNW-ZNS) and demonstrated its excellent liquidrepellent and $\mathrm{NH}_{3}$ gas sensing properties. The thorny nanowall structure consists of a vertical graphene nanowall (GNW) framework with numerous $\mathrm{ZnO}$ nanospikes (denoted as ZNS) branched on top of the nanowalls (Fig. 1a). Because of its unique reentrant and hierarchical characteristics, GNW-ZNS exhibits excellent liquid repellency toward various fluids without additional surface modification. By combining the advantages of gas sensing graphene and $\mathrm{ZnO}, \mathrm{GNW}-\mathrm{ZNS}$ can be successfully applied as an $\mathrm{NH}_{3}$ gas sensor at $\mathrm{RT}$ with reasonable sensitivity, and excellent selectivity and repeatability. Moreover, owing to the outstanding liquid repellency from the reentrant structure, the GNW-ZNS-based sensor also displays high stability with a good response even after severe bacterial contamination. This study offers a facile method for fabricating hierarchical and reentrant structures with robust hydrophobicity and liquid repellency and provides a unique strategy to engineer advanced gas sensors with reliable performance for extended applications.

\section{Results and discussion}

The hierarchical and reentrant GNW-ZNS was fabricated in three main steps (Fig. 1b), and the morphology of the obtained structures was characterized by scanning electron microscopy (SEM; Fig. 2a-c). Vertically aligned GNWs were first grown on $\mathrm{SiO}_{2} / \mathrm{Si}$ substrates by the microwave plasma enhanced chemical vapor deposition (MPECVD) method. As shown in Fig. 2a, nanowalls with a height of $\sim 3.2 \mu \mathrm{m}$ and edge length of $\sim 1.3 \mu \mathrm{m}$ uniformly stood, and were distributed on the $\mathrm{SiO}_{2} / \mathrm{Si}$ substrates. These interwoven nanowall sheets connected to each other, forming open and porous networks with an average pore size of $\sim 780 \mathrm{~nm}$. In the second step, a thin film of $\mathrm{ZnO}$ was deposited on the GNWs by magnetron sputtering (sample denoted as GNW-ZF). Due to the steric effect of the vertical nanowall structure with narrow spacing between the nanowalls, this $\mathrm{ZnO}$ layer could 

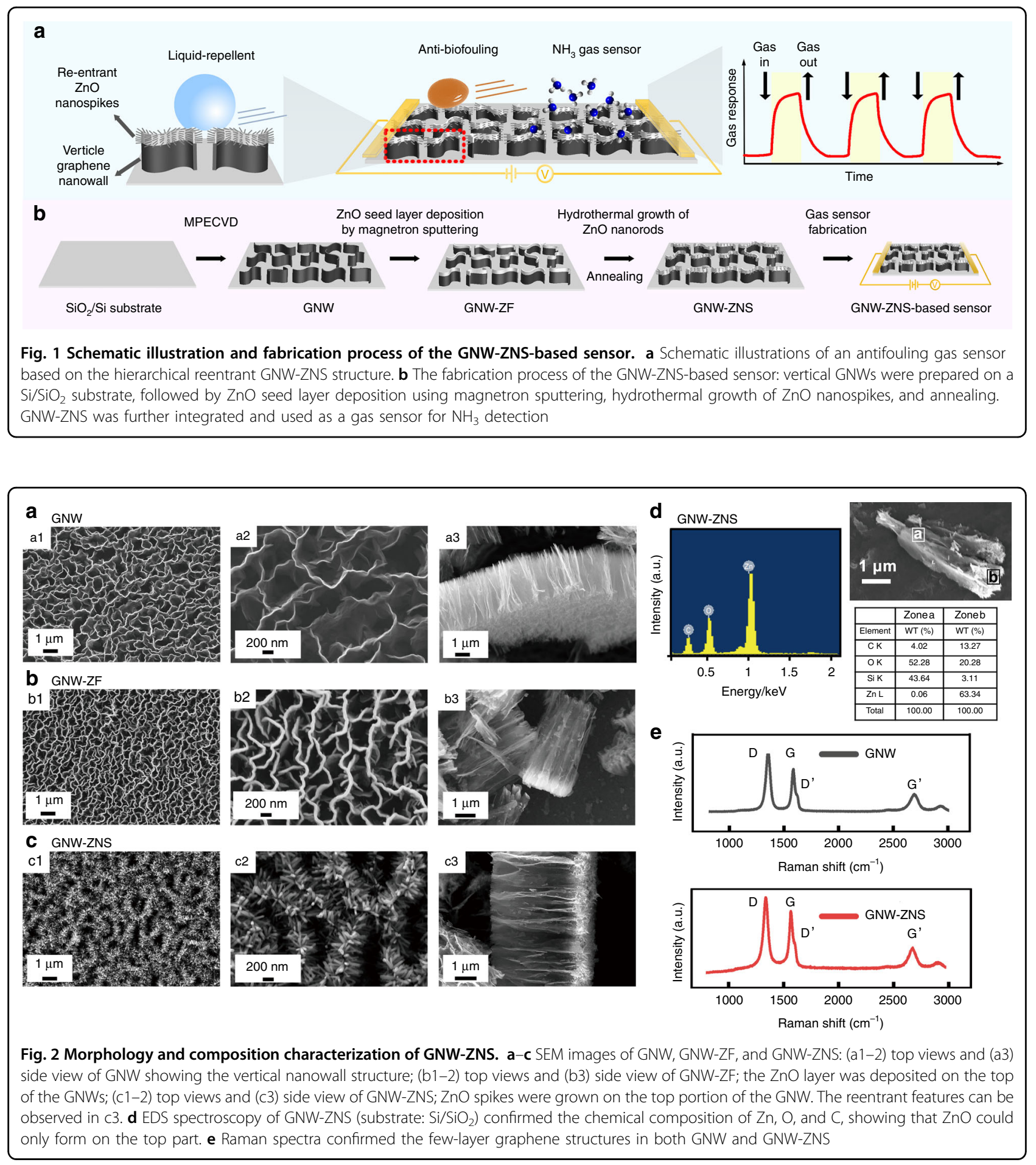

neither penetrate the bottom surface nor adhere to the sidewall surface of the nanowalls. Instead, only the top portion of the nanowalls was covered with $\mathrm{ZnO}$ (Fig. 2b). This $\mathrm{ZnO}$ layer served as the nucleation/seed layer for the subsequent growth of $\mathrm{ZnO}$ nanorods by a hydrothermal reaction in an aqueous solution of $\mathrm{Zn}\left(\mathrm{NO}_{3}\right)_{2}$ and diethylenetriamine. After the reaction, a reentrant thorny
$\mathrm{ZnO}$ /graphene hybrid nanowall structure was obtained, whose surface morphology after annealing at $150{ }^{\circ} \mathrm{C}$ is shown in Fig. 2c. In the final structure, numerous $\mathrm{ZnO}$ nanothorns with an average length of $220 \mathrm{~nm}$ and diameter of $60 \mathrm{~nm}$ were grown on top of the nanowalls. The resulting thorny nanowall-like hierarchical structure not only retained the characteristic structure of the vertical 
nanowalls from graphene, but also possessed an increased surface area and reentrant features because of the $\mathrm{ZnO}$ nanothorns introduced on the graphene top edge.

The chemical composition was investigated by energy dispersive $\mathrm{X}$-ray spectroscopy (EDS), confirming the existence of $\mathrm{C}, \mathrm{Zn}$, and $\mathrm{O}$ and showing that $\mathrm{ZnO}$ could only form on the top part of the GNW-ZNS (Fig. 2d). In addition, Raman spectra were recorded to further characterize the graphene structure in the GNW and GNW-ZNS (Fig. $2 \mathrm{e})$. For the GNW, the characteristic peaks of graphene, including the $\mathrm{G}$ band located at $\sim 1578 \mathrm{~cm}^{-1}$ and the $\mathrm{G}^{\prime}$ band (or 2D band) located at $2687 \mathrm{~cm}^{-1}$, were observed, demonstrating its few-layer graphene structure with an I $\left(G^{\prime}\right) /(G)$ intensity ratio of 0.43 (ref. ${ }^{29}$ ). The defect-related $\mathrm{D}$ band peak at $1347 \mathrm{~cm}^{-1}$ and the small $\mathrm{D}^{\prime}$ band peak at $1584 \mathrm{~cm}^{-1}$ indicated the presence of abundant defects, possibly from the graphene edges ${ }^{30,31}$. The Raman spectrum of GNW-ZNS was similar to that of GNW, suggesting that the graphene structure in GNW-ZNS remained intact without significant changes. In addition, because of the high controllability of the MPECVD, magnetron sputtering, and hydrothermal growth processes, the morphology of the obtained GNW-ZNS could also be controlled. For example, GNW-ZNS samples with different spacings could be obtained (Supplementary Fig. 1, Supplementary Table 1). In this study, GNW-ZNS with a moderate inter-nanowall spacing was deliberately chosen for subsequent study. This selection was made because an open and porous vertical nanowall structure with high surface area and sufficient spacing may provide favorable conditions for both antifouling and gas sensing.

The surface morphology and properties can determine wetting behaviors, and can influence gas sensing performance ${ }^{22,32}$. The wettability of the different surfaces was systematically studied. The contact angle (CA) with various liquids, including water, a bacterial suspension and blood, was measured for GNW-ZNS and compared with that of control samples, including GNW and GNW-ZF (Fig. 3a). To investigate the role of the reentrant feature in wettability, GNWs without overhanging suspended nanospikes but with uniformly distributed $\mathrm{ZnO}$ nanorods (denoted as GNW-ZNR) were fabricated and compared with GNW-ZNS. Briefly, GNW-ZNR was fabricated by multiple steps, including MPECVD of GNW, atomic layer deposition (ALD) of a $\mathrm{ZnO}$ seed layer, and $\mathrm{ZnO}$ nanorod branching by hydrothermal growth. The CA was determined using a Kruss DSA goniometer by placing a $4 \mu \mathrm{l}$ droplet onto the sample surface in air at RT (Fig. 3a) ${ }^{31}$. To show the difference in the liquid-to-solid adhesion characteristics of different samples, optical photos showing liquid droplets released from a height of $6 \mathrm{~cm}$ and impacting tilted surfaces (tilted angle $=2^{\circ}$ ) are also presented.

As shown in Fig. 3b-d, among all samples, GNW-ZNS displayed the highest hydrophobicity, with the highest water
CA of $150.4 \pm 6.6^{\circ}$. The control samples were less hydrophobic, with water CAs of $142.6 \pm 3.9^{\circ}$ for GNW, $130.9 \pm$ $3.0^{\circ}$ for GNW-ZF, and $125.2 \pm 13.3^{\circ}$ for GNW-ZNR. In addition, relatively high CAs $\left(>139^{\circ}\right)$ were obtained on GNW-ZNS for low-surface-tension liquids, including blood (surface tension $=51.6 \mathrm{mN} \mathrm{m}^{-1}$ at $298 \mathrm{~K}$ ) and a bacterial suspension (surface tension $=66.3 \mathrm{mN} \mathrm{m}^{-1}$ at $298 \mathrm{~K}$ ). Although all samples displayed hydrophobic properties, with CAs larger than $90^{\circ}$, the wetting behaviors of the surfaces differed considerably from those of GNW-ZNS and the other samples (Supplementary Table 2). The GNW-ZNS surface showed high liquid repellency, and all tested liquid drops quickly and readily bounced off the GNW-ZNS surface. In contrast, on the control samples, the liquids could not roll off and tended to become stuck. The control GNW, GNW-ZF, and GNW-ZNR surfaces were highly adhesive to the liquid drops. As shown in Fig. 3f, a $5 \mu$ water droplet remained firmly pinned even when the surfaces were tilted at $90^{\circ}$ or turned upside down. Our results showed that GNW-ZNS was highly anti-wetting, while the control samples exhibited a relatively high CA and high adhesion. In nature, similar wetting phenomena have been found, i.e., the "lotus effect" and the "rose petal effect" $^{\prime 33}$. Lotus leaves are superhydrophobic, and water droplets can easily roll away with an ultra-low sliding angle. Rose petals can support a water droplet with a large CA and high liquid affinity, and even if the petals are turned upside down, water droplets can remain on the surfaces. In this study, GNW-ZNS showed the "lotus effect", and the other control samples were similar to rose petals. The possible reason for this result is discussed as follows.

Graphene is commonly considered a hydrophobic material, and the water CA on graphene can be as high as $\sim 127^{\circ}$ (refs. ${ }^{34,35}$ ). The GNW material initially obtained by MPECVD displayed a relatively high water CA of $142.6^{\circ}$, possibly because the nano-gaps or nano-voids between the vertical nanowalls in GNW could trap air, leading to high CAs. Nevertheless, many of these pores in GNW were on the micrometer scale, and liquid may still penetrate these micro-gaps, causing strong adhesion. This wetting behavior of GNW was very similar to that of natural rose petals with micro-papillae, where liquid can impregnate larger grooves between micro-papillae but cannot enter smaller grooves. Our observations were also consistent with a previous report that flower-like fewlayer graphene nanoclusters on nanocone arrays show a high CA and ultrahigh adhesion ${ }^{36}$. Wettability is determined not only by morphological characteristics but also by material properties. Since the $\mathrm{ZnO}$ seed layer in GNWZF was relatively thin $(\sim 30 \mathrm{~nm})$ compared with that of GNW, the local roughness of GNW-ZF was altered slightly; therefore, the change in CA was mainly caused by the $\mathrm{ZnO}$. The surface free energy of $\mathrm{ZnO}$ is much higher than that of graphene ${ }^{34,37}$. Therefore, water may penetrate 


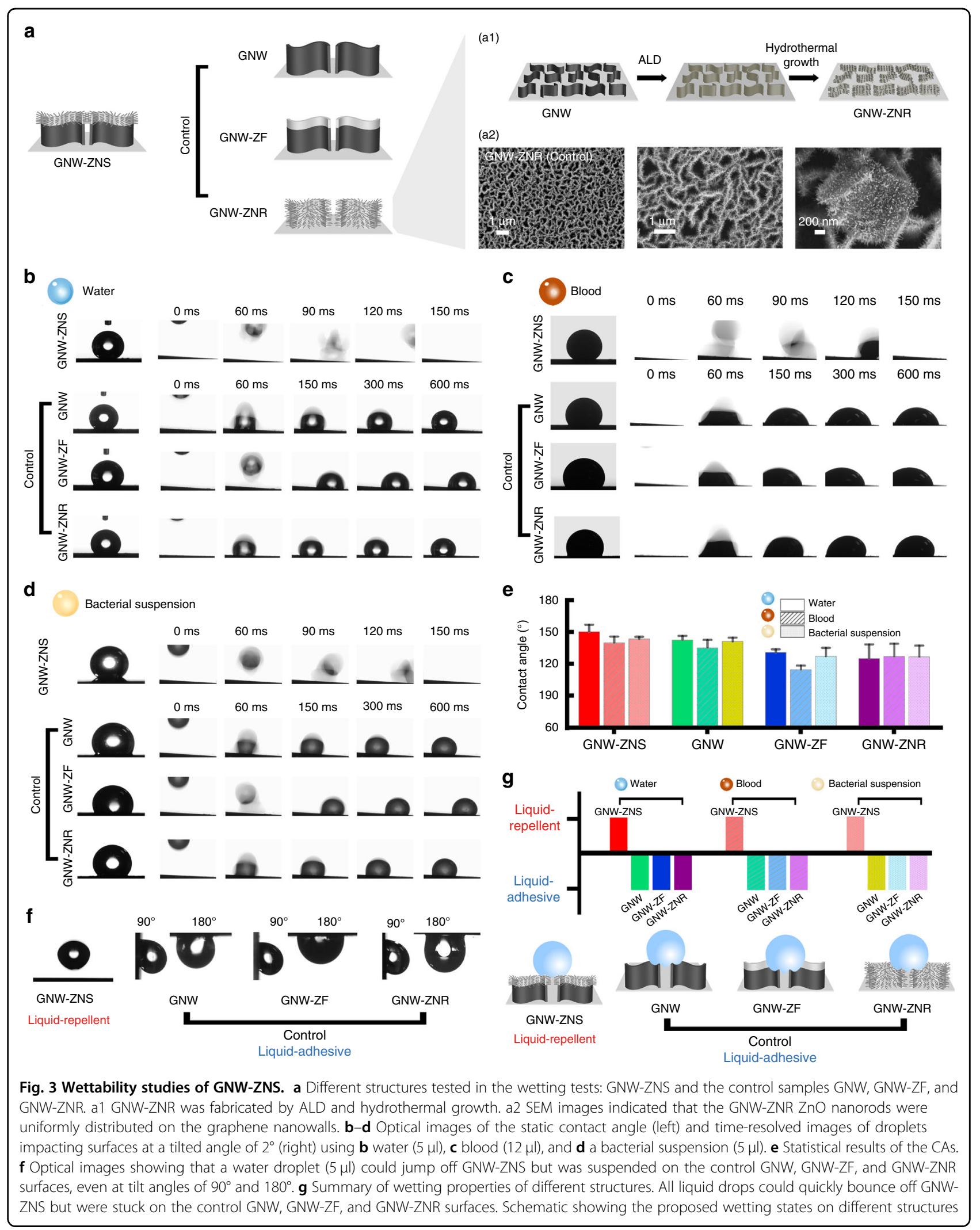


into the $\mathrm{ZnO}$ nanowall edge more deeply in GNW-ZF than in GNW, resulting in a decrease in CA, although $\mathrm{ZnO}$ was only deposited on the top fraction of the GNW. After hydrothermal growth of ZNS and moderate annealing, the hierarchical GNW-ZNS sample achieved excellent hydrophobicity with high CAs. Moreover, in contrast to GNW and GNW-ZF, the GNW-ZNS surface was highly liquid repellent (Fig. 3f), indicating that the hierarchical reentrant structure helped the hydrophobicity. Through hydrothermal growth, a large number of ZNS were grown on top, and accordingly, numerous small air pockets could form. Compared with GNW and GNW-ZF, the inter-nanowall spacing in GNW-ZNS decreased, and the initially exposed micro-sized pores or voids were trapped or blocked at the bottom due to the nanothorns introduced above. Therefore, the underlying nano- and micro-gaps could lock air more efficiently. In other words, the nano-air pockets created by the nanothorns on the upper part of the vertical nanowalls and the larger microvoids underneath could contribute to both entrapping air and helping GNW-ZNS suspend liquids, enabling liquid droplets to roll off the surface. GNWZNR, which had a hierarchical structure, also displayed a "rose petal effect" with high adhesion (Fig. 3g). In contrast with GNW-ZNS, the ZnO nanorods entirely covered the GNWs on both sides, and the graphene material was no longer exposed in GNW-ZNR. Since $\mathrm{ZnO}$ is less hydrophobic than graphene, the CAs of GNW-ZNR were lower, although there were nano-air pockets present in the material. Our results were in good agreement with previous studies, showing that hierarchical and reentrant structures are favorable for obtaining a stable hydrophobic surface with robust liquid-repellent properties $^{25,27,28}$.

GNW-ZNS consists of graphene and $\mathrm{ZnO}$, and both have been recognized as promising gas-sensitive materials for $\mathrm{NH}_{3}$ (refs. ${ }^{3,8}$ ). To explore the applicability of the GNW-ZNS structure as a gas sensor, the sample was further integrated into a device with silver wires attached to the surface using silver paste ${ }^{38}$. The device was placed in a sealed chamber (volume: $\sim 0.5 \mathrm{~L}$ ) and exposed to a series of premixed gases with different concentrations at a constant flow rate of $1000 \mathrm{sccm}$. The conductivity of the device was recorded at a bias voltage of $1 \mathrm{~V}$ using a source meter (Fig. 4a). The relative response (sensitivity) of the gas sensor was evaluated as $\left(I_{\text {air }-} I_{\text {gas }}\right) / I_{\text {air }} \times 100 \%$, and the recovery rate was evaluated as $\left(I_{\text {air-after }}-I_{\text {gas }}\right) /\left(I_{\text {air }}-I_{\text {gas }}\right) \times 100 \%$, where $I_{\text {air }}$ is the initial conductivity of the sensor in air, $I_{\text {gas }}$ is the minimum conductivity in the target gas, and $I_{\text {air-after }}$ is the stable conductivity recovered in air after treatment with the target gas. The response time was defined as the time for the current to achieve a $63.2 \%$ change with respect to the minimum conductivity ${ }^{39}$.
Figure $4 \mathrm{~b}$ shows representative relative response curves of GNW-ZNS- and GNW-based devices exposed to $\mathrm{NH}_{3}$ at RT. At a relatively low concentration of $\mathrm{NH}_{3}$, i.e., $150 \mathrm{ppm}$, obvious responses were observed for both the GNW-ZNS- and GNW-based devices, with minor differences. The average response was $2.11 \%$ for GNW-ZNS and $1.85 \%$ for GNW under $150 \mathrm{ppm} \mathrm{NH}_{3}$ at RT. The response increased with increasing $\mathrm{NH}_{3}$ concentration for both devices. However, different response characteristics could be observed at relatively high $\mathrm{NH}_{3}$ concentrations (Fig. 4c). The response increased significantly to $\sim 11.6 \%$ for the GNW-ZNS sensor under $\mathrm{NH}_{3}$ at $600 \mathrm{ppm}$, whereas the response of the GNW sensor only increased to $5.3 \%$. Moreover, the recovery rate was significantly decreased for the GNW sensor. As shown in Fig. 4b, the GNW sensor could not recover to its initial level, while good recovery was obtained with the GNW-ZNS sensor. The repeatability of the sensor was also investigated, and the results of the dynamic response for three repeated cycles are shown in Fig. $4 \mathrm{~d}$ in the case of detecting $\mathrm{NH}_{3}$ at a concentration of $400 \mathrm{ppm}$. The GNW-ZNS sensor displayed good reproducibility with stable recovery. In contrast, the sensing response of the GNW sensor was unstable without a consistent response trend under repeated cycles. The average response times estimated by the dynamic response curves were $\sim 73 \mathrm{~s}$ for the GNWZNS sensor and $52 \mathrm{~s}$ for the GNW sensor under $400 \mathrm{ppm}$ $\mathrm{NH}_{3}$. Note that the response time was overestimated due to the limit of our gas inlet configuration in the gas sensing system (Fig. 4e). The above results indicated that compared with the GNW sensor, the GNW-ZNS sensor could respond to $\mathrm{NH}_{3}$ over a wider ppm range with better sensitivity and reversibility despite a slightly longer response time. In addition, the selectivity of the sensors was tested against different analytes, including ethanol, acetone, and pure $\mathrm{O}_{2}$. Both sensors exhibited relatively low sensitivity toward various interferents, even at high concentrations, demonstrating their good selectivity toward $\mathrm{NH}_{3}$ (Fig. 5a).

Furthermore, the excellent liquid repellency of GNWZNS may offer the possibility of gas sensing with high stability even under severe conditions. To test this, the gas sensing performance of a biofouled GNW-ZNS sensor was evaluated. A GNW sensor with high liquid adhesion properties was also studied as a control. Fresh GNW-ZNS and GNW samples were placed on a tilted glass slide $\left(10^{\circ}\right)$, and a bacterial suspension was constantly deposited on the surfaces until a total of $2 \mathrm{ml}$ had been deposited. During this treatment, bacterial droplets could slide off the GNW-ZNS sample easily but tended to remain on the GNW sample (Fig. 5b). After rinsing and drying, the samples were further fabricated into sensors, and the gas sensing properties were evaluated. As shown in Fig. 5c, the GNW-ZNS sensor challenged by a bacterial 


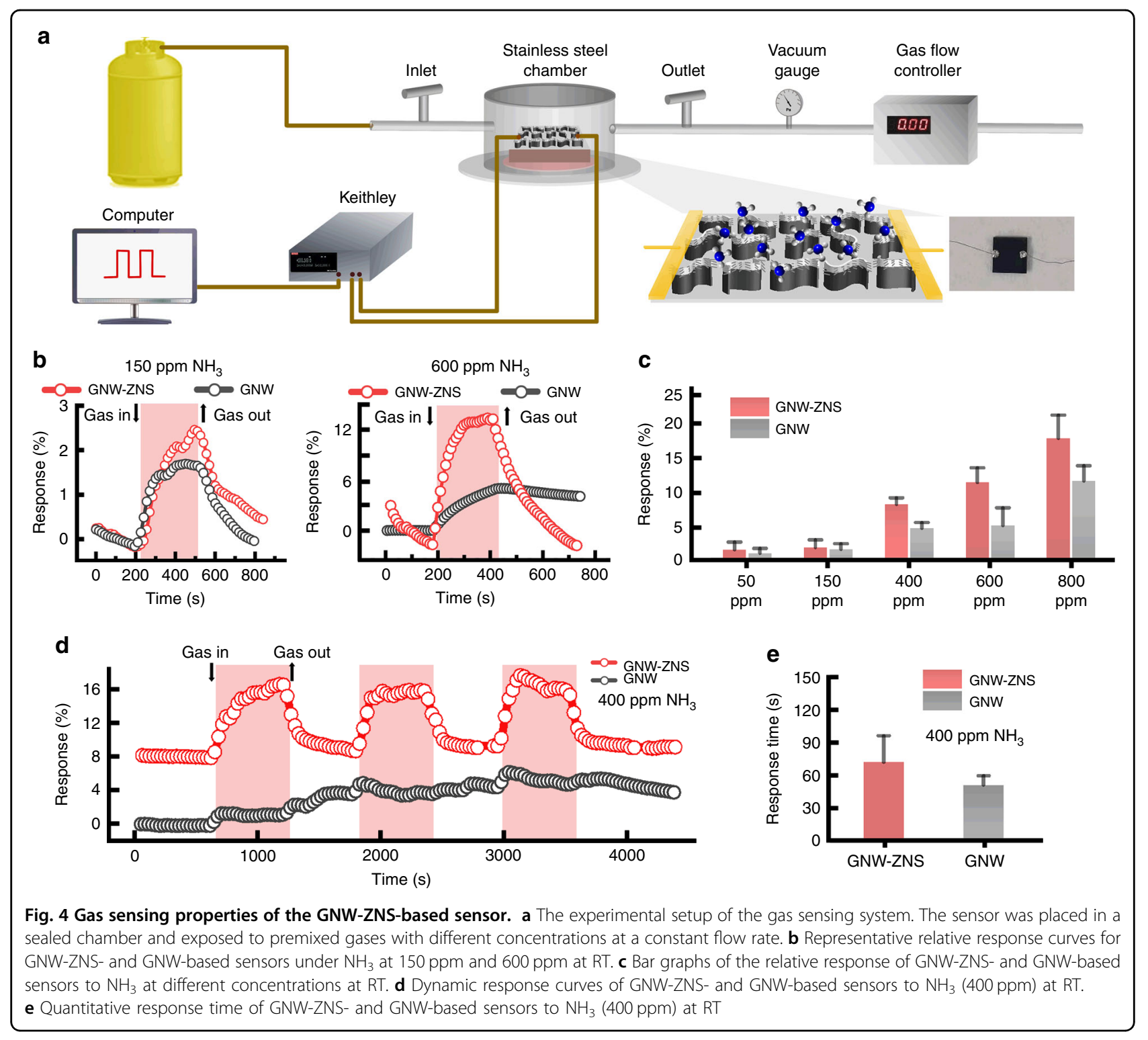

suspension still exhibited a stable response of $6.0 \%$ toward $400 \mathrm{ppm} \mathrm{NH}_{3}$, a response $\sim 70 \%$ that of an untreated GNW-ZNS sensor, which had an average response of 8.4\%. In contrast, the GNW sensor after bacterial fouling only showed a response of $1.7 \%$, indicating severe deterioration to only one-third the level of the untreated GNW sensors (4.9\% toward $400 \mathrm{ppm} \mathrm{NH}_{3}$ ). In addition, the GNW-ZNS sensor retained excellent recovery, while the GNW sensor could not readily recover its original conductivity. These results demonstrated that the GNWZNS sensor can display great stability under severe biofouling conditions, which is challenging to achieve with conventional gas sensors.

The gas sensing mechanism of graphene-based materials has been widely studied, yet it is not fully understood at present. In general, it is agreed that the detection of gas molecules is closely related to the charge transfer from the gas molecules to the sensitive surface, causing a change in the charge-carrier concentration and therefore a change in resistivity ${ }^{40}$. GNW-ZNS consists of graphene and $\mathrm{ZnO}$. Graphene normally exhibits p-type semiconducting behavior, and $\mathrm{ZnO}$ is an $\mathrm{n}$-type semiconductor ${ }^{6}$. The gas response characteristics of composites; however, vary in different studies since there are many controlling factors, such as morphology and component contents ${ }^{15,41-43}$. For example, in recent studies, rGO and $\mathrm{WO}_{3}$ (n-type) nanocomposites displayed p-type behavior toward $\mathrm{NH}_{3}$ detection ${ }^{41}$. Gas sensors fabricated from nanohybrids of $\mathrm{rGO}$ and $\mathrm{SnS}_{2}$ (ntype) exhibited transitions from $\mathrm{p}$-type to $\mathrm{n}$-type sensing behavior upon adjusting the content of rGO (ref. ${ }^{42}$ ). 


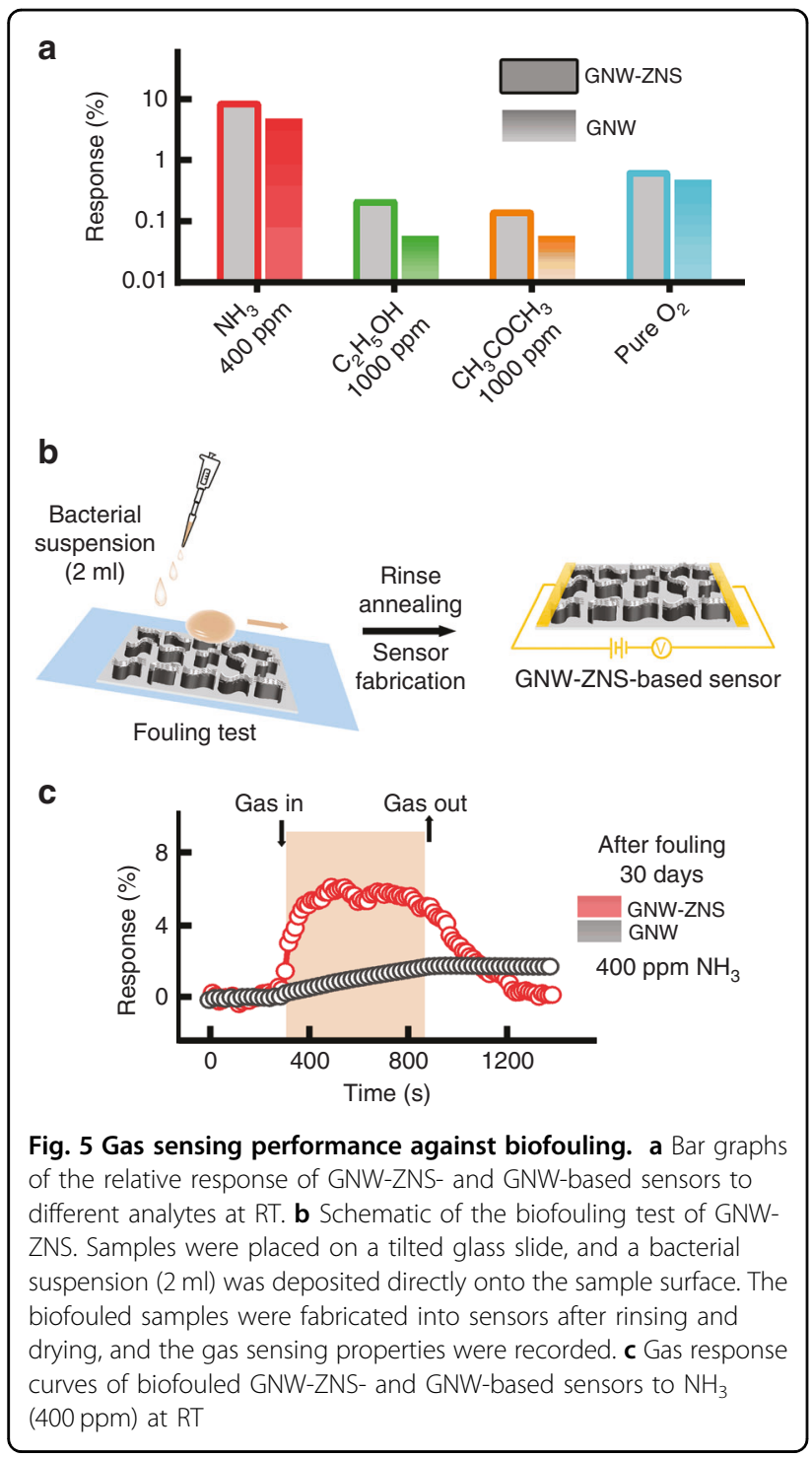

In this study, both the GNW-ZNS and GNW sensors exhibited p-type behavior ${ }^{15,44}$. It is possible that when GNW-ZNS and GNW were exposed to $\mathrm{NH}_{3}$ molecules, the $\mathrm{NH}_{3}$ molecules were absorbed on their surfaces, and electrons were transferred from the $\mathrm{NH}_{3}$ molecules to the structures due to the strong electron-donating property of the reducing gas $\mathrm{NH}_{3}$. Accordingly, the hole carrier concentration in both GNW-ZNS and GNW decreased, leading to reductions in their conductivity. The vertical nanowall structures in GNW-ZNS and GNW possessed a high surface area, allowing $\mathrm{NH}_{3}$ molecules to infiltrate the structures through plentiful inter-nanowall channels, thereby providing a good basis for gas detection ${ }^{7}$. The $\mathrm{p}-$ type behavior of GNW-ZNS was similar to that of GNW, suggesting that the response properties of GNW-ZNS were dominated by the GNW backbone, probably because the content of $\mathrm{ZnO}$ was relatively low. In addition, in
GNW-ZNS, ZNS were introduced purposely only at the top of the GNW, and the majority of the graphene was still exposed to contact with $\mathrm{NH}_{3}$ molecules, yielding a ptype sensing response.

Compared with the GNW sensor, the GNW-ZNS sensor not only showed a better response over a broader range toward $\mathrm{NH}_{3}$, but also better reproducibility and stability. The enhanced gas sensing response could be attributed to the hierarchical and reentrant features of GNW-ZNS. In GNW-ZNS, interfaces between different materials were created after depositing the $\mathrm{ZnO}$ layer, and $\mathrm{p}-\mathrm{n}$ heterojunctions could form between the graphene and $\mathrm{ZnO}$ in GNW-ZNS. The electron state in the depletion layers of heterojunctions will change when $\mathrm{NH}_{3}$ molecules are in contact with the interfaces, and the charge transfer in GNW-ZNS may be more active than that in GNW $\left(\right.$ refs. $\left.{ }^{15,16}\right)$. This behavior may explain why GNW-ZNS can display a good response even at relatively high concentrations of $\mathrm{NH}_{3}$. In contrast, the desorption process in the GNW sensor was more difficult. After adsorbing $\mathrm{NH}_{3}$ molecules, the sensor had difficulty recovering to its original state. In addition, the numerous ZNS significantly increased the specific surface area and may have resulted in more adsorption sites for $\mathrm{NH}_{3}$, which could also be favorable for gas sensing at high concentrations.

Furthermore, the hierarchical and reentrant features of GNW-ZNS provided excellent liquid repellency. When subjected to a severe biofouling challenge by direct deposition of a bacterial suspension, the material could resist contamination more effectively than the other samples and maintain excellent gas sensing ability. Sensors biofouled in relatively static conditions but for a longer time also demonstrated the superior stability of GNW-ZNS than GNW (please see Supplementary Fig. 2 for more details). Overall, acceptable and comparable sensitivity as well as high stability was observed for the GNW-ZNS sensor (Supplementary Table 3), which are desirable characteristics of gas sensors for extended application. It should also be mentioned that at the current stage, the influence of the steric effect of the nanospikes on the gas sensing properties of the GNWZNS sensor has not been fully clarified. Further work is required to examine this effect and determine the optimum GNW-ZNS structure in terms of both antifouling and gas sensing properties. Investigating the sensing characteristics of GNW-ZNS sensors with different inter-nanowall distances and comparing the sensing properties of GNW-ZNS with those of GNW-ZF (without hierarchical nanospikes) and GNW-ZNR (without exposed graphene) are possible ways to better study the underlying mechanisms.

\section{Conclusion}

In summary, we have demonstrated the effectiveness of a highly liquid-repellent hierarchical and reentrant graphene-based GNW-ZNS structure for the sensitive 
detection of $\mathrm{NH}_{3}$ at RT. GNW-ZNS features numerous branched ZNS on top of GNWs and can be fabricated by MPECVD, magnetron sputtering and hydrothermal growth. Owing to its unique reentrant features, the hierarchical GNW-ZNS exhibited excellent liquid repellency toward various fluids, such as bacterial suspensions, without surface modification. The GNW-ZNS structure could be further applied as an $\mathrm{NH}_{3}$ sensor with reasonable sensitivity over a broad ppm range. The vertical nanowall structure with plentiful inter-nanowall channels offered ample space for gas molecules to infiltrate the structure. The overhanging ZNS increased the surface area, possibly providing more adsorption sites and enhancing the charge transfer efficiency, resulting in a higher sensitivity than that of the GNW sensor. Moreover, the liquid repellency of GNW-ZNS made it possible for the sensor to maintain excellent gas sensing stability even after severe bacterial contamination. The anti-biofouling gas sensors described here provide a facile method to construct reentrant and hierarchical structures with desirable liquid-repellent properties without chemical surface modification. This research also opens new opportunities for designing highperformance gas sensors with resistance to biofouling, which could be applied in complicated bio-environments.

\section{Materials and methods}

The fabrication of GNW-ZNS mainly consisted of three steps. In the first step, GNWs were deposited on $\mathrm{Si} / \mathrm{SiO}_{2}$ substrates using the MPECVD method. Clean substrates (size: $5 \times 5 \times 0.5 \mathrm{~mm}$ or $10 \times 10 \times 1 \mathrm{~mm}$ ) were pretreated with $\mathrm{H}_{2}$ and $\mathrm{Ar}$ at $800{ }^{\circ} \mathrm{C}$ for $15 \mathrm{~min}$. Then, mixed $\mathrm{CH}_{4}$ (flow rate: $60 \mathrm{sccm}$ ) and $\mathrm{H}_{2}$ (flow rate: $10 \mathrm{sccm}$ ) were introduced as gas reactants and maintained at $800^{\circ} \mathrm{C}$ at a power of $1000 \mathrm{~W}$ for $20 \mathrm{~min}$. After cooling to RT under vacuum, GNW samples were obtained. Next, a $\mathrm{ZnO}$ layer with a thickness of $\sim 30 \mathrm{~nm}$ was prepared via a magnetron sputtering system (ZKDS VTC-300, China) under a vacuum of $2.0 \mathrm{~Pa}$ and $\mathrm{DC}$ power of $280 \mathrm{~W}$. By magnetron sputtering, the $\mathrm{ZnO}$ seed layer was deposited only on the top portion of the GNW. Finally, ZNS were grown on the $\mathrm{ZnO}$ seed layer using the hydrothermal growth method in a solution of hexamethylenetetramine $(25 \mathrm{mM})$ and $\mathrm{Zn}\left(\mathrm{NO}_{3}\right)_{2}(25 \mathrm{mM})$ at $90^{\circ} \mathrm{C}$ for $1.5 \mathrm{~h}$. After the hydrothermal reaction, the samples were rinsed with ethanol and distilled water several times, and annealed in a vacuum oven at $150^{\circ} \mathrm{C}$ for at least $24 \mathrm{~h}$. The control sample of GNWs with uniformly distributed $\mathrm{ZnO}$ nanorods (denoted as GNW-ZNR) was prepared by ALD and hydrothermal growth. By ALD, a ZnO seed layer with a thickness of $\sim 30 \mathrm{~nm}$ was deposited on the GNW sample. Then, $\mathrm{ZnO}$ nanorods were grown via a process similar to that used for GNW-ZNS. The GNWZNR sample was obtained after rinsing and annealing. Details regarding the fabrication process of GNW and GNW-ZNR can be found in our previous studies ${ }^{30,31}$.
The surface morphology of the obtained structures was characterized by SEM (Zeiss Supra 60). The composition and structure of the samples were investigated by EDS (Oxford Instrument) and Raman spectroscopy (Renishaw inVia Reflex spectrometer system). Wetting properties were studied by measuring the CAs and performing liquid Escherichiaimpacting tests with a goniometer measuring system (Kurss, DSA 15). Static CAs were measured by placing a $4 \mu \mathrm{l}$ droplet onto the sample surface at RT. Different liquids, including deionized water, blood (whole blood of sheep, RuiTe Company, China), and bacterial suspensions (Escherichia coli, ATCC25922, Rui Bio, $\sim 10^{7}$ colony forming units), were tested as probe liquids. At least three measurements were repeated for each sample to obtain a reliable result. Liquid impacting tests were conducted by recording free-falling liquid droplets (water and bacterial droplets: $5 \mu \mathrm{l}$; blood droplet: $12 \mu \mathrm{l}$ ) impacting a tilted sample surface (tilted angle $=2^{\circ}$ ) from a height of $6 \mathrm{~cm}$.

The gas sensing performance was evaluated using a homemade measurement setup. A sample (substrate size: $5 \times 5 \mathrm{~mm}$ ) was integrated into a device with silver wires attached at two different points on its surface. The contacts to the wires were simply made using conductive silver paste (SCP, No. SCP50G). In a typical gas sensing test, the device was placed in a sealed chamber (volume: $\sim 0.5 \mathrm{~L}$ ). The electric characteristics of the device were monitored by recording the conductivity continuously at a voltage of $1 \mathrm{~V}$ using a source meter (Keithley 2400 or 2601). After a steady current was measured in air, premixed gases were then introduced into the chamber for a certain time at a constant flow rate of $1000 \mathrm{sccm}$ by a gas flow controller (QC-1S, Beijing Municipal Institute of Labour Protection, China). The concentrations of premixed gases, including $\mathrm{NH}_{3}$ and ethanol, were calculated according to the injected amount in the gas container and further calibrated using commercial gas sensors (PLT811NH3, PLT840, Anchuangjia, China).

The relative response (sensitivity) of the gas sensor was evaluated as $\left(I_{\text {air }}-I_{\text {gas }}\right) / I_{\text {air }} \times 100 \%$, and the recovery rate was evaluated as $\left(I_{\text {air-after }}-I_{\text {gas }}\right) /\left(I_{\text {air }}-I_{\text {gas }}\right) \times 100 \%$, where $I_{\text {air }}$ is the initial conductivity of the sensor in air, $I_{\text {gas }}$ is the minimum conductivity in the target gas, and $I_{\text {air-after }}$ is the stable conductivity recovered in air after treatment with the target gas. The response time was defined as the time for the current to achieve a $63.2 \%$ change with respect to the minimum conductivity ${ }^{39}$. The reported response was the average value for at least four individual devices.

The stability of the sensors was evaluated by contaminating the samples with a bacterial suspension. Fresh samples were placed on a tilted glass slide (tilted angle $=$ $\left.10^{\circ}\right)$, and E. coli bacterial suspensions $\left(\sim 10^{7}\right.$ colony forming units, $2 \mathrm{ml}$ in total) were deposited directly on the sample surfaces. The samples were then rinsed with distilled water and ethanol three times. After drying, the 
samples were fabricated into sensors, and the gas sensing properties were then recorded at RT. Additional biofouling tests on a group of GNW-ZNS and GNW sensors were also performed to compare the sensing performance before and after static contamination. Pretested GNWZNS and GNW sensors were briefly biofouled, and the sensing properties were tested after bacterial contamination. Bacterial suspension droplets $(20 \mu \mathrm{l})$ were directly deposited onto the surfaces, and the samples were incubated at $37^{\circ} \mathrm{C}$ for $20 \mathrm{~min}$. The bacterial suspension was then removed, and the samples were rinsed and dried. The electrode was reapplied, and the gas sensing performance was retested.

\section{Acknowledgements \\ The authors acknowledge the financial support from the National Natural Science Foundation of China (grant nos. 61771498, 61901535, 51805556, 51705543, and 31900954), the Youth Teachers' Development Program of Sun Yat-sen University (nos. 18lgpy21 and 18lgpy18), the Science and Technology Planning Project of Guangdong Province for Industrial Applications (no. 2017B090917001), the Guangdong Province Key Area R\&D Program (grant no. 2018B030332001), the Science and Technology Program of Guangzhou, China (grant no. 201907010038), the Guangdong Basic and Applied Basic Research Foundation (grant no. 2019A1515012087), and the Open Research Fund of State Key Laboratory of Bioelectronics, Southeast University, for support.}

\section{Author details}

'State Key Laboratory of Optoelectronic Materials and Technologies, School of Electronics and Information Technology, Guangdong Province Key Laboratory of Display Material and Technology, Sun Yat-Sen University, Guangzhou 510006, China. ${ }^{2}$ School of Materials Science and Energy Engineering, Foshan University, Foshan 528000, China. ${ }^{3}$ The First Affiliated Hospital of Sun Yat-Sen University, Guangzhou 510080, China

\section{Author contributions}

T.H., J.W., S.X., B.L., Chengduan Yang, Cheng Yang, and Y.X. carried out the experimental work; T.H., J.W., Cheng Yang, and N.H. performed the data analysis; Y.Z. and X.X. supervised the study; and T.H., Y.Z., and X.X. drafted the manuscript. All authors gave final approval for publication.

\section{Conflict of interest}

The authors declare that they have no conflict of interest.

Supplementary information accompanies this paper at https://doi.org/ 10.1038/s41378-020-0151-5.

Received: 28 November 2019 Revised: 23 February 2020 Accepted: 3 March 2020

Published online: 13 July 2020

\section{References}

1. Singh, E., Meyyappan, M. \& Nalwa, H. S. Flexible graphene-based wearable gas and chemical sensors. ACS Appl. Mater. Interfaces 9, 34544-34586 (2017).

2. Wang, T. et al. A review on graphene-based gas/vapor sensors with unique properties and potential applications. Nano-Micro Lett. 8, 95-119 (2016).

3. Wetchakun, $K$. et al. Semiconducting metal oxides as sensors for environmentally hazardous gases. Sens. Actuators B Chem. 160, 580-591 (2011).

4. Schedin, F. et al. Detection of individual gas molecules adsorbed on graphene. Nat. Mater. 6, 652-655 (2007).

5. Leenaerts, O., Partoens, B. \& Peeters, F. M. Adsorption of $\mathrm{H}(2) \mathrm{O}, \mathrm{NH}(3), \mathrm{CO}, \mathrm{NO}$ (2), and NO on graphene: a first-principles study. Phys. Rev. B 77, 125416 (2008).

6. Dan, Y., Lu, Y., Kybert, N. J., Luo, Z. \& Johnson, A. T. C. Intrinsic response of graphene vapor sensors. Nano Lett. 9, 1472-1475 (2009).
7. Yavari, F. et al. High sensitivity gas detection using a macroscopic threedimensional graphene foam network. Sci. Rep. 1, 166 (2011).

8. Vikrant, K. Kumar, V. \& Kim, K. H. Graphene materials as a superior platform for advanced sensing strategies against gaseous ammonia. J. Mater. Chem. A $\mathbf{6}$, 22391-22410 (2018)

9. Yi, G. C., Wang, C. R. \& Park, W. I. ZnO nanorods: synthesis, characterization and applications. Semiconductor Sci. Technol. 20, S22-S34 (2005).

10. Wang, C. et al. Reduced graphene oxide decorated with CuO-ZnO heterojunctions: towards high selective gas-sensing property to acetone. J. Mater. Chem. A 2, 18635-18643 (2014).

11. Hahn, Y.-B., Ahmad, R. \& Tripathy, N. Chemical and biological sensors based on metal oxide nanostructures. Chem. Commun. 48, 10369-10385 (2012).

12. Meng, R., Lu, X., Ingebrandt, S. \& Chen, X. Adsorption of gas molecules on graphene-like $\mathrm{ZnO}$ nanosheets: the roles of gas concentration, layer number, and heterolayer. Adv. Mater. Interfaces 4, 1700647 (2017).

13. Devan, R. S., Patil, R. A., Lin, J.-H. \& Ma, Y.-R. One-dimensional metal-oxide nanostructures: recent developments in synthesis, characterization, and applications. Adv. Funct. Mater. 22, 3326-3370 (2012).

14. Zhang, D., Yin, N. \& Xia, B. Facile fabrication of $\mathrm{ZnO}$ nanocrystalline-modified graphene hybrid nanocomposite toward methane gas sensing application. J. Mater. Sci. Mater. Electron. 26, 5937-5945 (2015).

15. Xia, Y. et al. Confined formation of ultrathin $\mathrm{ZnO}$ nanorods/reduced graphene oxide mesoporous nanocomposites for high-performance room-temperature $\mathrm{NO}_{2}$ sensors. ACS Appl. Mater. Interfaces 8, 35454-35463 (2016).

16. Tai, H. et al. ZnO Nanoparticles/reduced graphene oxide bilayer thin films for improved $\mathrm{NH}_{3}$-sensing performances at room temperature. Nanoscale Res. Lett. 11, 130 (2016).

17. Chatterjee, S. G., Chatterjee, S., Ray, A. K. \& Chakraborty, A. K. Graphene-metal oxide nanohybrids for toxic gas sensor: a review. Sens. Actuators B Chem. 221 1170-1181 (2015)

18. Lin, Q., Li, Y. \& Yang, M. Tin oxide/graphene composite fabricated via a hydrothermal method for gas sensors working at room temperature. Sens. Actuators B Chem. 173, 139-147 (2012).

19. Harper, L. A., Flesch, T. K. \& Wilson, J. D. Ammonia emissions from broiler production in the San Joaquin Valley 1. Poult. Sci. 89, 1802-1814 (2010).

20. Bixler, G. D. \& Bhushan, B. Biofouling: lessons from nature. Philos. Trans. A Math Phys. Eng. Sci. 370, 2381-2417 (2012).

21. Pechook, $\mathrm{S}$. et al. Bioinspired passive anti-biofouling surfaces preventing biofilm formation. J. Mater. Chem. B 3, 1371-1378 (2015).

22. $\mathrm{Wu}$, J. et al. $3 \mathrm{D}$ superhydrophobic reduced graphene oxide for activated $\mathrm{NO}_{2}$ sensing with enhanced immunity to humidity. J. Mater. Chem. A 6, 478-488 (2018).

23. Tuteja, A., Choi, W., Mabry, J. M., McKinley, G. H. \& Cohen, R. E. Robust omniphobic surfaces. Proc. Natl Acad. Sci. USA 105, 18200-18205 (2008).

24. Tripathy, A. et al. Enhancing the bactericidal efficacy of nanostructured multifunctional surface using an ultrathin metal coating. Langmuir 33, 12569-12579 (2017)

25. Hang, T. et al. Self-cleaning ultraviolet photodetectors based on tree crownlike microtube structure. Adv. Mater. Interfaces 6, 1801251 (2019).

26. Tuteja, A. et al. Designing superoleophobic surfaces. Science 318, 1618-1622 (2007).

27. Bielinski, A. R. et al. Rational design of hyperbranched nanowire systems for tunable superomniphobic surfaces enabled by atomic layer deposition. ACS Nano 11, 478-489 (2017)

28. Hoshian, S., Jokinen, V., Somerkivi, V., Lokanathan, A. R. \& Franssila, S. Robust superhydrophobic silicon without a low surface-energy hydrophobic coating. ACS Appl. Mater. Interfaces 7, 941-949 (2015).

29. Malard, L. M., Pimenta, M. A., Dresselhaus, G. \& Dresselhaus, M. S. Raman spectroscopy in graphene. Phys. Rep. Rev. Sect. Phys. Lett. 473, 51-87 (2009).

30. Zhang, Y. et al. Growth direction manipulation of few-layer graphene in the vertical plane with parallel arrangement. Carbon 56, 103-108 (2013).

31. Hang, T. et al. Hierarchical graphene/nanorods-based $\mathrm{H}_{2} \mathrm{O}_{2}$ electrochemical sensor with self-cleaning and anti-biofouling properties. Sens. Actuators $B$ Chem. 289, 15-23 (2019).

32. Some, S. et al. Highly sensitive and selective gas sensor using hydrophilic and hydrophobic graphenes. Sci. Rep. 3, 1868 (2013).

33. MacGregor-Ramiasa, M. N. \& Vasilev, K. Questions and answers on the wettability of nano-engineered surfaces. Adv. Mater. Interfaces 4, 1700381 (2017).

34. Wang, S. R., Zhang, Y., Abidi, N. \& Cabrales, L. Wettability and surface free energy of graphene films. Langmuir 25, 11078-11081 (2009).

35. Taherian, F., Marcon, V., van der Vegt, N. F. A. \& Leroy, F. What is the contact angle of water on graphene? Langmuir 29, 1457-1465 (2013). 
36. Tian, S. B. et al. Robust adhesion of flower-like few-layer graphene nanoclusters. Sci. Rep. 2, 511 (2012)

37. Ghatak, J., Huang, J. H. \& Liu, C. P. Derivation of the surface free energy of $\mathrm{ZnO}$ and GaN using in situ electron beam hole drilling. Nanoscale 8, 634-640 (2016).

38. Yang, J. et al. Wearable temperature sensor based on graphene nanowalls. Rsc Adv. 5, 25609-25615 (2015).

39. Mao, S. et al. Tuning gas-sensing properties of reduced graphene oxide using tin oxide nanocrystals. J. Mater. Chem. 22, 11009-11013 (2012).

40. Jin, L. F., Chen, W. G. \& Zhang, Y. Application of graphene hybrid materials in fault characteristic gas detection of oil-immersed equipment. Front. Chem. $\mathbf{6}$, 399 (2018).
41. Jeevitha, $\mathrm{G}$. et al. Porous reduced graphene oxide $(\mathrm{rGO}) / \mathrm{WO}_{3}$ nanocomposites for the enhanced detection of $\mathrm{NH} 3$ at room temperature. Nanoscale Adv. 1, 1799-1811 (2019).

42. Huang, $\mathrm{Y}$. et al. Ultrasensitive room temperature ppb-level $\mathrm{NO}_{2}$ gas sensors based on $\mathrm{SnS}_{2} / \mathrm{rGO}$ nanohybrids with $\mathrm{P}-\mathrm{N}$ transition and optoelectronic visible light enhancement performance. J. Mater. Chem. C 7, 8616-8625 (2019).

43. Schuett, F., Postica, V., Adelung, R. \& Lupan, O. Single and networked ZnO-CNT hybrid tetrapods for selective room-temperature high-performance ammonia sensors. Acs Appl. Mater. Interfaces 9, 23107-23118 (2017).

44. Lu, G., Ocola, L. E. \& Chen, J. Reduced graphene oxide for room-temperature gas sensors. Nanotechnology 20, 445502 (2009). 